\title{
Physician and Surrogate Agreement with Assisted Dying and Continuous Deep Sedation in Advanced Dementia in Switzerland
}

\author{
Andrea Jutta Loizeau ${ }^{\mathrm{a}-\mathrm{c}}$ Simon M. Cohen ${ }^{\mathrm{c}}$ Susan L. Mitchell ${ }^{\mathrm{c}, \mathrm{d}}$ \\ Nathan Theilla, b, e Stefanie Eicher ${ }^{a, b}$ Mike Martin $^{a, b, f}$ Florian Riese $^{a, e}$ \\ aUniversity Research Priority Program "Dynamics of Healthy Aging," University of Zurich, Zurich, Switzerland;

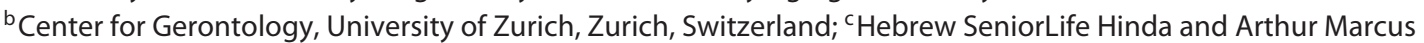 \\ Institute for Aging Research, Boston, MA, USA; ${ }^{d}$ Department of Medicine, Beth Israel Deaconess Medical Center,

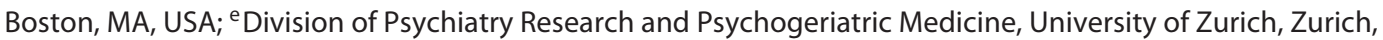 \\ Switzerland; ' Department of Psychology, University of Zurich, Zurich, Switzerland
}

\section{Keywords}

Dementia · Assisted dying · Sedation · End of life ·

Decision-making

\begin{abstract}
Background: Assisted dying and continuous deep sedation (CDS) are controversial practices. Little is known about the perceptions of physicians and surrogates about these practices for patients with advanced dementia. Objectives: To describe and compare physician and surrogate agreement with the use of assisted dying and CDS in advanced dementia. Design, Setting, Subjects: Physicians $(n=64)$ and surrogates $(n=168)$ of persons with advanced dementia were recruited as part of a randomized controlled trial in Switzerland that tested decision support tools in this population. Methods: At baseline, the participants were asked about their agreement with assisted dying and CDS in advanced dementia using the following response options: "completely agree," "somewhat agree," "somewhat disagree," "com-
\end{abstract}

pletely disagree," and "do not know." Multivariable logistic regressions compared the likelihood that surrogates versus physicians would completely or somewhat agree (vs. completely or somewhat disagree) with these practices. Results: The physicians and surrogates, respectively, had a mean age (SD) of 50.6 years (9.9) and 57.4 years (14.6); $46.9 \%(n=30 / 64)$ and $68.9 \%(n=115 / 167)$ were women. A total of $20.3 \%(n=$ $13 / 64)$ of the physicians and $47.0 \%(n=79 / 168)$ of the surrogates agreed with assisted dying in advanced dementia. Surrogates were significantly more likely to agree with this practice than physicians (adjusted odds ratio, 3.87; 95\% Cl: $1.94,7.69)$. With regard to CDS, $51.6 \%(n=33 / 64)$ of the physicians and $41.9 \%(n=70 / 169)$ of the surrogates agreed with this practice, which did not differ significantly between the groups. Conclusions: The surrogates were more agreeable to considering assisted dying in the setting of advanced dementia than the physicians, and about half of the participants in both groups reported CDS to be an appropriate option for this population.

(c) 2019 S. Karger AG, Basel

\section{KARGER}

(c) 2019 S. Karger AG, Basel

E-Mail karger@karger.com

www.karger.com/ndd
Andrea Jutta Loizeau, $\mathrm{PhD}$

Hebrew SeniorLife Hinda and Arthur Marcus Institute for Aging Research 1200 Centre Street

Boston, MA 02131 (USA)

E-Mail andrealoizeau@hsl.harvard.edu 


\section{Introduction}

Dementia afflicts more than 50 million people worldwide and is one of the most common causes of death in Switzerland $[1,2]$. In advanced dementia, patients have profound cognitive and functional deficits, and they experience clinical complications that may cause discomfort and a poor quality of life [3-5].

Assisted dying is a controversial practice that has been used in certain contexts for patients with life-limiting disease [6]. Assisted dying is when a physician either administers drugs that cause a person to die (i.e., euthanasia) or provides lethal drugs for self-administration (i.e., physician-assisted suicide) [7]. In the case of euthanasia, the physician takes an active role in completing the request of the patient incapable of completing the final act, for instance, by administering a lethal injection. In assisted suicide, the physician solely makes lethal means available to the patient, but the completion of the final act is left to the patient. Currently, physicianassisted dying is legal in only a few states in the USA as well as in a limited number of countries, including Switzerland $[6,8,9]$. However, only the Netherlands, Belgium, and Luxembourg allow assisted dying for people with advanced dementia [10]. This request must be made in an advance directive by a patient with dementia when decision-making capacity is still intact. Only limited research exists on the perceptions of physicians and surrogates about the use of assisted dying in advanced dementia [11-15].

Another controversial practice for terminally ill patients is continuous deep sedation (CDS) until death, a last-resort palliative practice to alleviate suffering [16, 17]. Medications are administered until death to lower the level of consciousness and relieve refractory symptoms of patients with a life expectancy of typically less than 2 weeks [18]. To date, CDS has predominantly been used for advanced cancer patients who are experiencing extreme physical suffering in their final weeks of life $[16,19]$. CDS has rarely been used in advanced dementia, a condition predominantly characterized by cognitive impairments, with some, but typically less, physical suffering than metastatic cancer [3, $5,20]$.

To better understand the perceptions of physicians and surrogates about the use of assisted dying and CDS in advanced dementia, we analyzed baseline data from a randomized controlled trial, the DEMentia FACT boxes (DEMFACT) Study, conducted in the Swiss German region of Switzerland. The objec- tives of this report were to (1) describe physician and surrogate agreement with the use of assisted dying and CDS in advanced dementia, and (2) compare the agreement with the use of these practices in these two groups.

\section{Subjects and Methods}

\section{Data Source}

The data were retrieved from baseline assessments made in the DEMFACT Study, conducted between June 2016 and October 2016 in the Swiss German region of Switzerland. DEMFACT was a randomized controlled trial that evaluated FACT box decision support tools for treatment decision-making regarding advanced dementia among 64 physicians and 168 surrogates (relatives of dementia patients, $n=100$; professional guardians, $n=68)$ who were randomized to either an intervention arm ( $n$ $=114)$ or a control arm $(n=118)$ [21]. The participants in the intervention and control arms were mailed written questionnaires at baseline and 1 month later that included questions about the use of treatments for advanced dementia patients based on hypothetical scenarios. At the 1-month follow-up, the intervention participants received the decision support tools, whereas the control participants were given no additional information.

The main DEMFACT Study compared changes in prespecified outcomes between the baseline and follow-up assessments in the intervention arm relative to the control arm. In this report, combined data from the baseline questionnaires in both arms (i.e., before receiving the decision support tools in the intervention arm) were analyzed to describe agreement with the use of continuous sedation and assisted dying in advanced dementia. The baseline questionnaires were completed between June 2, 2016, and July 31, 2016.

\section{Population}

To understand the impact of the DEMFACT intervention on various decision-makers, the participants included physicians as well as surrogates (relatives of dementia patients and professional guardians) who were potentially responsible for the care of advanced dementia patients. Physicians were identified from the mailing lists of the Swiss Association for Palliative Medicine, Care and Support, and the Swiss Geriatric Medicine Society. Surrogates were identified either through the Alzheimer Association of the canton of Zurich or from the Swiss Association of Professional Guardians mailing list. The detailed recruitment procedure is described elsewhere [21]. Eligible participants were mailed a consent form, which they were asked to sign and return to the research team.

\section{Data Elements}

Data collection was procedurally identical across physician and surrogate participants. All variables in this report were collected at the DEMFACT baseline assessment using a written questionnaire sent and returned by mail (approx. $60 \mathrm{~min}$ to complete). 
Table 1. Characteristics of the physicians and surrogates $(n=232)$

\begin{tabular}{|c|c|c|}
\hline Characteristics & $\begin{array}{l}\text { Physicians } \\
(n=64)\end{array}$ & $\begin{array}{l}\text { Surrogates } \\
(n=168)\end{array}$ \\
\hline Mean age $\pm S D^{a}$, years & $50.6 \pm 9.9$ & $57.4 \pm 14.6$ \\
\hline Age $>55$ years ${ }^{b}$ & $21(32.8)$ & $91(54.5)$ \\
\hline Female $^{\mathrm{a}}$ & $30(46.9)$ & $115(68.9)$ \\
\hline \multicolumn{3}{|l|}{ Nationality $^{\mathrm{a}}$} \\
\hline Swiss & $44(72.1)$ & $157(94.6)$ \\
\hline German & $13(21.3)$ & $7(4.2)$ \\
\hline Other & $4(6.6)$ & $2(1.2)$ \\
\hline \multicolumn{3}{|l|}{ Religion $^{\mathrm{a}}$} \\
\hline Protestant or catholic & $44(68.8)$ & $108(65.5)$ \\
\hline Other & $9(14.1)$ & $11(6.7)$ \\
\hline No religion & $10(15.6)$ & $38(23.0)$ \\
\hline Refused to answer & $1(1.6)$ & $8(4.8)$ \\
\hline \multicolumn{3}{|l|}{ Education $^{\mathrm{a}}$} \\
\hline High school or higher & $64(100)$ & $161(97.0)$ \\
\hline \multicolumn{3}{|l|}{ Any prior major treatment } \\
\hline decision in dementia ${ }^{a, c}$ & $58(90.6)$ & $42(25.3)$ \\
\hline
\end{tabular}

Values denote $n$ (\%) unless specified otherwise. ${ }^{\text {a }}$ The total number of missing values by characteristic was: age, $n=1$; female, $n=1$; nationality, $n=5$; religion, $n=3$; education, $n=2$; and any prior major treatment decision, $n=2 .{ }^{\mathrm{b}}$ Median age. ${ }^{\mathrm{c}}$ Decisionmakers reported whether they had or had not previously made any major decision about the use of antibiotics and/or artificial hydration for a person with advanced dementia.

The outcomes were participants' agreement with the use of assisted dying and CDS in advanced dementia. Agreement with assisted dying was ascertained using the following question: "The current legislation prohibits physician-assisted suicide (assisted dying) for persons lacking the capacity to consent. This prevents persons with advanced dementia from accessing the services of assisted dying organizations. Independent of the current legal situation, would you personally support the use of assisted suicide (one form of physician-hastened death) for persons with advanced dementia?" Agreement with the use of CDS was measured as follows: "At the end of life, it is possible to use medications, administered until death, to relieve the symptoms of a person with advanced dementia and put them into a permanent, artificial sleep (continuous sedation). Would you personally support the use of continuous sedation for persons with advanced dementia?" For both questions, the participants were asked to select one of the following response options: "completely agree," "somewhat agree," "somewhat disagree," "completely disagree," or "do not know."

Other participant data assessed at baseline included demographics (age, gender, nationality [Swiss, German, and other], religion [Protestant or Catholic, other religion, no religion, and refused to answer], and educational level [ $\geq$ high school vs. other]) and whether participants had previously decided about the use of antibiotics and/or artificial hydration for a person with advanced dementia.
Analysis

The analyses were performed using $\mathrm{R}$ version 3.3.2 (Boston, MA, USA). Means with standard deviations (SDs) and frequencies describe continuous and categorical variables, respectively.

Logistic regression was used to examine the association between decision-maker type (surrogate vs. physician) and agreement with the following practices in advanced dementia (outcomes): (1) assisted dying and (2) CDS. For each practice, the outcome was dichotomized as "agree" (i.e., "completely agree" and "somewhat agree") versus "disagree" (i.e., "completely disagree" and "somewhat disagree") and excluded "do not know" responses. Covariates considered a priori to be possibly associated with supporting the use of assisted dying or CDS [13] included participants' demographic characteristics (age [dichotomized at the median], gender, and religion [dichotomized as "no religion" vs. "any," excluding "refused to answer"]) and prior decisions about the use of antibiotics and/or artificial hydration in advanced dementia (dichotomized as "any prior major treatment decision" vs. "none"). Bivariable analyses examined the unadjusted associations between each covariate and the outcome. Variables associated with the outcome at $p<0.10$ in the unadjusted analyses were entered into a multivariable model. The final adjusted model included those variables significantly associated with the outcome at $p<0.05$. Adjusted odds ratios (AORs) with 95\% confidence intervals (CIs) were computed. A sensitivity analysis examined whether the association differed as a function of subgroup (relatives vs. proxies), since perceptions might not have been homogeneous across subgroups.

\section{Results}

\section{Subject Characteristics}

Of the 3,860 individuals approached for participation, $254(6.6 \%)$ contacted the research team indicating their willingness to participate, and all were eligible for enrollment. Prior to study completion, 15 participants (5.9\%) stopped responding to e-mails and/or phone calls (physicians, $n=9 / 74$ [12.2\%]; surrogates, $n=6 / 180$ [3.3\%]) and 7 participants $(2.8 \%)$ withdrew (physicians, $n=1 / 74$ [1.4\%]; surrogates, $n=6 / 180$ [3.3\%]). The final sample included the remaining 64 physicians and 168 surrogates.

The baseline characteristics of the physicians and surrogates are shown in Table 1 . The physicians' mean age (SD) was 50.6 years $(9.9) ; 46.9 \%(n=30 / 64)$ were women and $72.1 \%(n=44 / 61)$ were Swiss. The surrogates' mean age (SD) was 57.4 years $(14.6) ; 68.9 \%(n=115 / 167)$ were women and $94.6 \%(n=157 / 166)$ were Swiss. A total of $68.8 \%(n=44 / 64)$ of the physicians and $65.5 \%(n=$ $108 / 165)$ of the surrogates reported being either Protestant or Catholic. A total of $90.6 \%(n=58 / 64)$ of the physi- 
Table 2. Association between decision-maker type and agreement with the use of assisted dying in advanced dementia

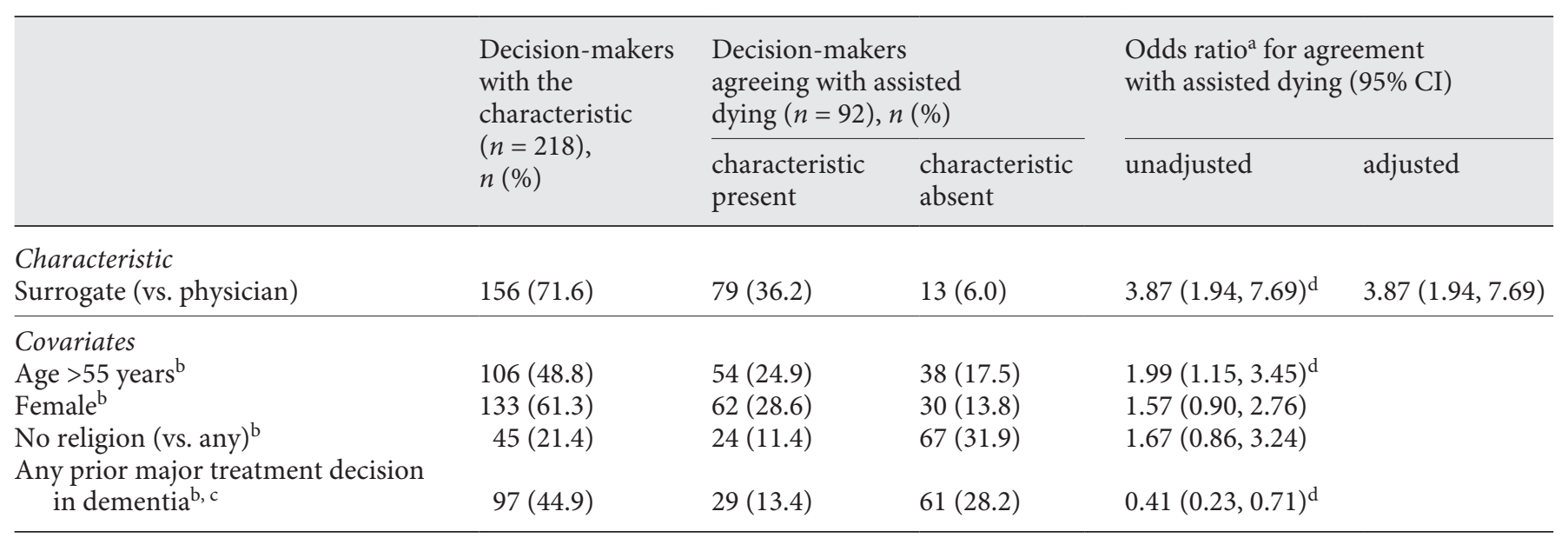

Decision-makers: $42.2 \%(n=92 / 218)$ agreed with the use of assisted dying in advanced dementia. ${ }^{\text {a }}$ Logistic regression was used in all analyses, and unadjusted and adjusted odds ratios were computed. ${ }^{\mathrm{b}}$ The total number of missing values was: age, $n=1 ;$ female, $n=$ 1 ; no religion, $n=8$ (missing values, $n=3$; refused, $n=5$ ); and any prior major treatment decision, $n=2 .{ }^{\mathrm{c}}$ Decision-makers reported whether they had or had not previously made any major decision about the use of antibiotics and/or artificial hydration for a person with advanced dementia. ${ }^{\mathrm{d}}$ Variables that were significant at $p<0.10$ in the bivariable analyses and entered into the multivariable model.

cians had previously made a major treatment decision for a person with advanced dementia, whereas only $25.3 \%$ $(n=42 / 166)$ of the surrogates had ever made such a decision.

\section{Agreement with Assisted Dying}

The distribution of physician responses about the use of assisted dying in advanced dementia was as follows: completely agree, $4.7 \%(n=3 / 64)$; somewhat agree, $15.6 \%$ $(n=10 / 64)$; somewhat disagree, $26.6 \%(n=17 / 64)$; completely disagree, $50.0 \%(n=32 / 64)$; and do not know, $3.1 \%(n=2 / 64)$. The distribution among the surrogates was: completely agree, $20.2 \%(n=34 / 168)$; somewhat agree, $26.8 \%(n=45 / 168)$; somewhat disagree, $23.2 \%$ ( $n=$ $39 / 168)$; completely disagree, $22.6 \%(n=38 / 168)$; and do not know, $7.1 \%(n=12 / 168)$.

In the unadjusted analyses, participant variables associated with agreement with assisted dying at $p<0.10$ were: age $>55$ years; any prior major treatment decision in dementia; and being a surrogate (vs. a physician) (Table 2). After multivariable adjustment, only being a surrogate (vs. a physician) remained significantly associated with a higher likelihood of agreeing with the use of assisted dying (AOR, 3.87; 95\% CI: 1.94, 7.69). When analyzing surrogate subgroups, agreement was significantly more likely among relatives than among proxies (34.6 vs. 16.0\%; AOR, 2.00; 95\% CI: 1.04, 3.83) (Appendix, Table 1).

End-of-Life Practices in Advanced Dementia

\section{Agreement with CDS}

The distribution of physician responses about the use of CDS in advanced dementia was as follows: completely agree, $20.3 \%(n=13 / 64)$; somewhat agree, $31.3 \%(n=$ $20 / 64)$; somewhat disagree, $31.3 \%(n=20 / 64)$; completely disagree, $6.3 \%(n=4 / 64)$; and do not know, $10.9 \%(n=$ $7 / 64)$. The distribution among the surrogates was: completely agree, $12.6 \%(n=21 / 167)$; somewhat agree, $29.3 \%$ $(n=49 / 167)$; somewhat disagree, $28.7 \%(n=48 / 167)$; completely disagree, $18.6 \%(n=31 / 167)$; and do not know, $10.8 \%(n=18 / 167)$.

The only covariate associated with a greater likelihood of supporting the use of CDS at $p \leq 0.10$ was "no religion" (vs. "any") (Table 3). After adjusting for religion, being a surrogate (vs. a physician) remained not significantly associated with agreement with the use of CDS (AOR, 0.69; 95\% CI: 0.36, 1.29). When analyzing surrogate subgroups, agreement was significantly more likely among relatives than among proxies (34.9 vs. $12.1 \%$; AOR, 2.82; 95\% CI: 1.41, 5.64) (Appendix, Table 2).

\section{Discussion}

In this report, a minority of the physicians (20\%) and about half of the surrogates (47\%) supported the use of assisted dying for patients with advanced dementia. Sur- 
Table 3. Association between decision-maker type and agreement with the use of continuous deep sedation in advanced dementia

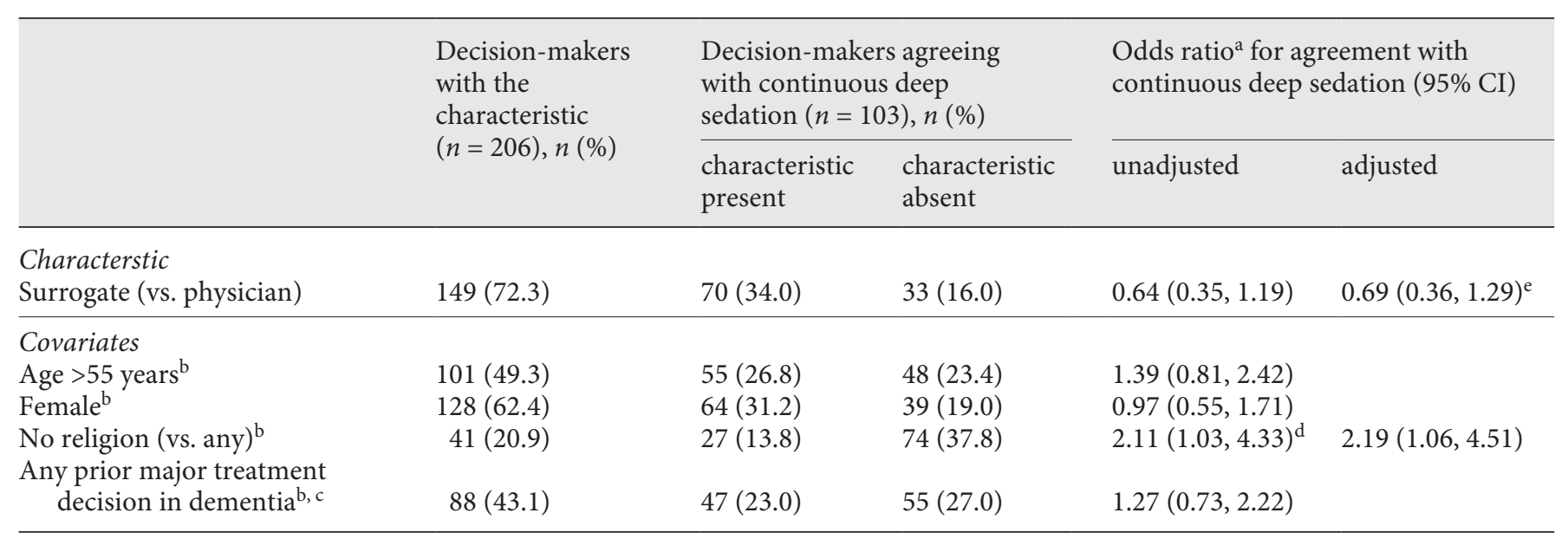

Decision-makers: $50.0 \%(n=103 / 206)$ agreed with the use of continuous deep sedation in advanced dementia. ${ }^{a}$ Logistic regression was used in all analyses, and unadjusted and adjusted odds ratios were computed. ${ }^{\mathrm{b}}$ The total number of missing values was: age, $n=1$; female, $n=1$; no religion, $n=10$ (missing values, $n=2$; refused, $n=8$ ); and any prior major treatment decision, $n=2 .{ }^{\mathrm{c}}$ Decision-makers reported whether they had or had not previously made any major decision about the use of antibiotics and/or artificial hydration for a person with advanced dementia. ${ }^{\mathrm{d}}$ The only variable that was significant at $p<0.10$ in the bivariable analyses and entered into the multivariable model. ${ }^{\mathrm{e}}$ After adjusting for religion, the association between being a surrogate and agreement with the use of continuous deep sedation remained not significant.

rogates were significantly more likely to agree with the use of this practice than physicians. About half of the physicians $(52 \%)$ and a slightly lower proportion of the surrogates (42\%) agreed with the use of CDS for these patients, which was not a significant difference between the groups.

This study confirms and extends the limited existing research on the perceptions of physicians and surrogates about assisted dying in advanced dementia [11-15]. The use of assisted dying has increased in countries where it is legal (The Netherlands and Belgium) over the last decade, but much less frequently for patients with dementia than for those with terminal illnesses that do not impact decision-making abilities $[6,10,22]$. Prior research has shown that most physicians are opposed to the use of this practice in dementia [12-15]. Irrespective of the presence of an advance directive, physicians are reluctant to perform physician-assisted suicide for patients lacking decision-making capacity [12]. A Dutch study on 1,456 physicians found that physicians were more willing to perform assisted dying for cancer patients with intact cognition (85\%) than for advanced dementia patients who had requested euthanasia in an advance directive prepared when they were still capable of making medical decisions (33\%) [14]. Similar proportions were found in a recent Canadian study on 136 physicians [15]. Notably, this study also showed that agreement increased to $71 \%$ when the hypothetical scenario specified that the dementia patients were in their last days of life. One chief concern is that preferences change over time [23], and that patients who imagine a future state with advanced dementia as one not worth living, once in that state, may appear to still retain a desire to live. Although surrogates may share this concern, their more favorable view of the use of assisted dying may be driven by a relatively greater aversion to their loved one experiencing the poor quality of life, suffering, and indignities of advanced dementia [11, 13]. The greater likelihood of surrogates agreeing to assistance in dying is due to the fact that their participation, unlike physicians, is not required to complete the act.

Our findings build on the very limited data on the use of CDS in advanced dementia $[3,15,20]$, and they reveal that there is no consensus among either physicians or surrogates about the appropriateness of using CDS in this population. This finding corroborates qualitative research showing that both groups of key decision-makers have mixed feelings about the use of this practice among terminally ill patients $[24,25]$. To date, CDS has predominantly been used and studied in advanced cancer, a condition where decision-making capacity remains intact and the dying process is most commonly 
accompanied by intractable physical pain and suffering $[16,19]$. In contrast to these patients, advanced dementia patients cannot participate in decision-making or report the source of their discomfort due to their serious cognitive impairments [5]. Therefore, it may be challenging for physicians to assess the intensity of a patient's suffering, making it difficult to justify the use of CDS in advanced dementia. Furthermore, pain and agitation in advanced dementia are often caused by other medical complications, such as infections, and may be effectively controlled with standard palliative medications $[3,4]$.

This study has several limitations that merit discussion. First, our participation rate was low, and thus our findings cannot be generalized to eligible nonparticipants. Second, generalizability is also limited to the Swiss German region of Switzerland and to hypothetical situations. The perceptions about end-of-life practices may vary in other regions of Switzerland and in real-life situations. In addition, it cannot be ruled out that physicians unaffiliated with associations promoting palliative care may hold different views on end-of-life practices. Third, the questions may not have been detailed enough to detect nuances in participants' perceptions [26]. It is likely that participants' opinions would have been different if they had been given more information, such as a statement indicating that the patient had made a written request for assisted dying before losing decisionmaking capacity. Likewise, providing participants with background information and definitions of different end-of-life practices such as physician-assisted suicide and euthanasia in the case of incompetent patients would likely have altered the responses. Lastly, the statistical power may have been insufficient to detect significant differences in secondary outcomes, notably differences in perceptions about CDS. Future work should replicate these findings in a clinical cluster-randomized trial.

This study expands upon the limited existing literature on the perceptions of physicians and surrogates about the use of assisted dying and CDS for patients with advanced dementia. Physicians were more opposed to the use of assisted dying than were the surrogates, and about half of the participants in both groups perceived CDS as an appropriate option in advanced dementia. In general, there is a clear need to improve end-of-life care in this patient population. However, due to the practical, ethical, and legal issues associated with performing assisted dying and CDS for patients who lack decisionmaking capacity, it remains unclear whether these prac-

End-of-Life Practices in Advanced Dementia tices would help accomplish this goal. Early goals of care discussions can promote decisions that align with the patient's preferences and the use of standard palliative medications $[27,28]$.

\section{Acknowledgments}

The investigators wish to thank the data management team (Daniel Gfeller and Kathrin Heiniger), the organizations' staff who helped with recruitment, Marius C. Vollberg for statistical guidance, and the participants who gave their time to this study.

\section{Statement of Ethics}

The ethics commission of the canton of Zurich approved the study's conduct (KEK-ZH-No. 2015-0626). All participants provided written informed consent. The authors have no ethical conflicts to disclose.

\section{Disclosure Statement}

The authors have no conflicts of interest to declare.

\section{Funding Sources}

This work was supported by the following grants: the Research in Palliative Care funding program (PC 22/14), which was offered jointly by the Swiss Academy of Medical Sciences, the Stanley Thomas Johnson Foundation, and the Gottfried and Julia Bangerter-Rhyner Stiftung; the Swiss National Science Foundation (P1ZHP3_171747 to A.J.L.); and the United States National Institutes of Health - National Institute on Aging (NIH-NIA R01 AG032982, NIH-NIA R01 AG043440, and NIH-NIA K24AG033640 to S.L.M.).

\section{Author Contributions}

F.R. and A.J.L. had full access to all data in the study and take responsibility for the integrity of the data and the accuracy of the data analyses. Study concept and design: A.J.L., N.T., S.E., M.M., and F.R.; acquisition of data: A.J.L., S.M.C., S.L.M., N.T., S.E., M.M., and F.R.; analyses and interpretation of data: A.J.L., S.M.C., S.L.M., N.T., S.E., M.M., and F.R.; drafting of manuscript: A.J.L., S.M.C., S.L.M., and F.R.; critical revision of manuscript for important intellectual content: A.J.L., S.M.C., S.L.M., N.T., S.E., M.M., and F.R.; statistical analyses: A.J.L., S.M.C., S.L.M., N.T., M.M., and F.R.; administrative, technical, or material support: A.J.L., S.E., and F.R.; and study supervision: A.J.L., N.T., S.E., M.M., and F.R. 


\section{Appendix}

Table 1. Association between being a relative versus a proxy and agreement with the use of assisted dying in advanced dementia

\begin{tabular}{|c|c|c|c|c|}
\hline & $\begin{array}{l}\text { Surrogates with the } \\
\text { characteristic } \\
(n=156), n(\%)\end{array}$ & \multicolumn{2}{|c|}{$\begin{array}{l}\text { Surrogates agreeing with assisted } \\
\text { dying }(n=79), n(\%)\end{array}$} & $\begin{array}{l}\text { Odds ratio }{ }^{\mathrm{a}} \text { for } \\
\text { agreement with assisted } \\
\text { dying }(95 \% \mathrm{CI}) \\
\text { adjusted }^{\mathrm{b}}\end{array}$ \\
\hline $\begin{array}{l}\text { Characteristic } \\
\text { Relative (vs proxv) }\end{array}$ & $94(603)$ & $54(346)$ & $25(16.0)$ & $200(1.04,3.83)$ \\
\hline
\end{tabular}

Surrogates: $50.6 \%(n=79 / 156)$ agreed with the use of assisted dying in advanced dementia. ${ }^{\text {a }}$ Logistic regression was used in all analyses, and unadjusted and adjusted odds ratios were computed. ${ }^{\mathrm{b}}$ After accounting for age, gender, religion, and prior decisions about antibiotics/artificial hydration use, no variables were significant at $p<0.10$ in the bivariable analyses, except for the association between being a relative versus proxy and agreement with the use of assisted dying in advanced dementia.

Table 2. Association between being a relative versus a proxy and agreement with the use of continuous deep sedation in advanced dementia

Surrogates with the characteristic

$(n=149), n(\%)$
Surrogates agreeing with

continuous deep sedation $(n=70), n(\%)$

characteristic present characteristic absent
Odds ratio ${ }^{\text {a }}$ for agreement with continuous deep sedation $(95 \% \mathrm{CI})$

adjusted $^{\mathrm{b}}$

\section{Characteristic}

Relative (vs. proxy)

$92(61.7)$

$52(34.9)$

$18(12.1)$

$2.82(1.41,5.64)$

Surrogates: $47.0 \%(n=70 / 149)$ agreed with the use of continuous deep sedation in advanced dementia. ${ }^{\text {a }}$ Logistic regression was used in all analyses, and unadjusted and adjusted odds ratios were computed. ${ }^{b}$ After accounting for age, gender, religion, and prior decisions about antibiotics/artificial hydration use, no variables were significant at $p<0.10$ in the bivariable analyses, except for the association between being a relative versus proxy and agreement with the use of continuous deep sedation in advanced dementia.

\section{References}

1 Federal Statistical Office FSO [Internet]. Cause of death statistics: death and its main causes in Switzerland, 2015 [accessed 2018 Apr 26]. Available from: https://www. bfs.admin.ch/bfsstatic/dam/assets/3742832/ master.

2 World Health Organization [Internet]. The epidemiology and impact of dementia: current state and future trends [accessed 2018 Apr 26]. Available from: http://www. who.int/mental_health/neurology/dementia/dementia_thematicbrief_epidemiology. pdf.

3 Hendriks SA, Smalbrugge M, Hertogh CM, van der Steen JT. Dying with dementia: symptoms, treatment, and quality of life in the last week of life. J Pain Symptom Manage. 2014 Apr;47(4):710-20.

4 Mitchell SL, Teno JM, Kiely DK, Shaffer ML, Jones RN, Prigerson HG, et al. The clinical course of advanced dementia. N Engl J Med. 2009 Oct;361(16):1529-38.
5 van der Steen JT, Deliens L, Koopmans RT, Onwuteaka-Philipsen BD. Physicians' perceptions of suffering in people with dementia at the end of life. Palliat Support Care. 2017 Oct;15(5):587-99.

6 Emanuel EJ, Onwuteaka-Philipsen BD, Urwin JW, Cohen J. Attitudes and practices of euthanasia and physician-assisted suicide in the United States, Canada, and Europe. JAMA. 2016 Jul;316(1):79-90.

7 Radbruch L, Leget C, Bahr P, Müller-Busch C, Ellershaw J, de Conno F, et al.; Board Members of EAPC. Euthanasia and physician-assisted suicide: a white paper from the European Association for Palliative Care. Palliat Med. 2016 Feb;30(2):104-16.

8 Swiss Academy of Medical Sciences [Internet]. Medical ethical guidelines: management of dying and death [accessed 2018 Jun 7]. Available from: https://www.samw.ch/en/ Publications/Medical-ethical-Guidelines. html.
9 Li M, Watt S, Escaf M, Gardam M, Heesters A, O'Leary G, et al. Medical assistance in dying - implementing a hospital-based program in Canada. N Engl J Med. 2017 May;376(21): 2082-8.

10 Neil F [Internet]. Assisted dying practice in Benelux: whitepaper 1 [accessed 2018 Apr 15]. Available from: http://www.dyingforchoice.com/resources/fact-files/assisted-dying-benelux-whitepaper-1.

11 Tomlinson E, Spector A, Nurock S, Stott J. Euthanasia and physician-assisted suicide in dementia: a qualitative study of the views of former dementia carers. Palliat Med. 2015 Sep;29(8):720-6.

12 Kouwenhoven PS, Raijmakers NJ, van Delden JJ, Rietjens JA, van Tol DG, van de Vathorst S, et al. Opinions about euthanasia and advanced dementia: a qualitative study among Dutch physicians and members of the general public. BMC Med Ethics. 2015 Jan; 16(7):7. 
13 Tomlinson E, Stott J. Assisted dying in dementia: a systematic review of the international literature on the attitudes of health professionals, patients, carers and the public, and the factors associated with these. Int J Geriatr Psychiatry. 2015 Jan;30(1):10-20.

14 Bolt EE, Snijdewind MC, Willems DL, van der Heide A, Onwuteaka-Philipsen BD. Can physicians conceive of performing euthanasia in case of psychiatric disease, dementia or being tired of living? J Med Ethics. 2015 Aug;41(8): 592-8.

15 Bravo G, Rodrigue C, Arcand M, Downie J, Dubois MF, Kaasalainen S, et al. Quebec physicians' perspectives on medical aid in dying for incompetent patients with dementia. Can J Public Health. 2018 Dec;109(5-6):729-39.

16 Miccinesi G, Rietjens JA, Deliens L, Paci E, Bosshard G, Nilstun T, et al.; EURELD Consortium. Continuous deep sedation: physicians' experiences in six European countries. J Pain Symptom Manage. 2006 Feb;31(2): 122-9.

17 Ziegler S, Schmid M, Bopp M, Bosshard G, Puhan MA. Continuous deep sedation until death - a Swiss death certificate study. J Gen Intern Med. 2018 Jul;33(7):1052-9.

18 Cherny NI, Radbruch L; Board of the European Association for Palliative Care. European Association for Palliative Care (EAPC) recommended framework for the use of sedation in palliative care. Palliat Med. 2009 Oct; 23(7):581-93.
19 McCarthy EP, Phillips RS, Zhong Z, Drews RE, Lynn J. Dying with cancer: patients' function, symptoms, and care preferences as death approaches. J Am Geriatr Soc. 2000 May;48(5 Suppl):S110-21.

20 Anquinet L, Rietjens JA, Vandervoort A, van der Steen JT, Vander Stichele R, Deliens L, et al. Continuous deep sedation until death in nursing home residents with dementia: a case series. J Am Geriatr Soc. 2013 Oct;61(10): 1768-76.

21 Loizeau AJ, Theill N, Cohen SM, Eicher S, Mitchell SL, Meier S, et al. Fact Box decision support tools reduce decisional conflict about antibiotics for pneumonia and artificial hydration in advanced dementia: a randomized controlled trail. Age Ageing. 2019 Jan;48(1): 67-74.

22 Dierickx S, Deliens L, Cohen J, Chambaere K. Euthanasia for people with psychiatric disorders or dementia in Belgium: analysis of officially reported cases. BMC Psychiatry. 2017 Jun;17(1):203.
23 Emanuel EJ, Fairclough DL, Emanuel LL. Attitudes and desires related to euthanasia and physician-assisted suicide among terminally ill patients and their caregivers. JAMA. 2000 Nov;284(19):2460-8.

24 Bruinsma SM, Rietjens JA, Seymour JE, Anquinet $\mathrm{L}$, van der Heide A. The experiences of relatives with the practice of palliative sedation: a systematic review. J Pain Symptom Manage. 2012 Sep;44(3):431-45.

25 Ziegler S, Merker H, Schmid M, Puhan MA The impact of the inpatient practice of continuous deep sedation until death on healthcare professionals' emotional well-being: a systematic review. BMC Palliat Care. 2017 May;16(1):30.

26 Magelssen M, Supphellen M, Nortvedt P, Materstvedt LJ. Attitudes towards assisted dying are influenced by question wording and order: a survey experiment. BMC Med Ethics. 2016 Apr;17(1):24

27 Mitchell SL, Palmer JA, Volandes AE, Hanson LC, Habtemariam D, Shaffer ML. Level of care preferences among nursing home residents with advanced dementia. Pain Symptom Manage. 2017 Sep;54(3): 340-5.

28 Loizeau AJ, Shaffer ML, Habtemariam DA, Hanson LC, Volandes AE, Mitchell SL. Association of prognostic estimates with burdensome interventions in nursing home residents with advanced dementia. JAMA Intern Med. 2018 Jul;178(7):922-9. 